\title{
KINEMATICS AND DYNAMICS
}

\author{
G. A. Gurzadian \\ (Branch of Byurakan Astrophysical Observatory, U.S.S.R.)
}

I would probably not be mistaken in saying that for the first time in the practice of solid scientific meetings the problem of the dynamics of planetary nebulae has become a subject of broad and sufficiently many-sided discussion. At the same time, I am inclined to estimate the successes achieved at the present symposium as more than modest. However, there have been no sensations. To the contrary of diminishing the importance and value of the reports made here, I only want to underline the fact that despite its attractiveness, the problem of the dynamics of planetary nebulae remains as before one of the difficult and complicated fields of theoretical and practical astrophysics. It cannot be solved without the cooperation of contiguous branches of science, particularly gas dynamics, magnetohydrodynamics and others. These are new components without which the physics of planetary nebulae has, nevertheless, managed. At the same time, almost all of the physics of planetary nebulae is comprised in the problem of the dynamics. Finally, the problems of the dynamics of planetary nebulae cannot be solved in an approach detached from the problem of the origin of planetary nebulae themselves and also from the origin and development of their nuclei. In this sense, the dynamics of planetary nebulae is becoming to a certain degree a cosmogonic science.

After these remarks of a general character, permit me to dwell on some concrete questions of the dynamics of planetary nebulae. To my mind, the following problems are of particular interest.

(1) The problem of internal motions of planetary nebulae. Taking into consideration the small magnitudes of the velocities of these motions, it is quite obvious that great care should be taken in applying delicate and complex methods of observation. We have seen successful examples of the application of such methods in the reports of Osterbrock, Münch, Courtès and Sheglov. It is expected that the field of application will expand. It is especially necessary to raise the sensitivity of these methods. However, the absence of small-scale (chaotic) motions inside nebulae should not be considered finally proven.

(2) The problem of planetary nebulae having two envelopes. As far as I know, only two works have been devoted to the problem of double-enveloped planetary nebulae that of the Gurzadian (1962) and of Minin (1958). In both works an attempt was made to show that the origin of the second (external) envelope is an unavoidable consequence of the peculiar activity of the pressure of Ly- $\alpha$ radiation in the nebulae. The

Osterbrock and O'Dell (eds.), Planetary Nebulae, 450-455. C, I.A.U. 
peculiarity lies in the fact that at a definite stage of development, i.e. expansion of the planetary nebula, $L y-\alpha$-radiation pressure strongly and spasmodically increases, which leads to the breaking off of parts of the external layers of the nebula from its primary mass. The process of breaking off may take place only once in the life of a nebula as its optical thickness in the frequencies of Lyman continuum radiation can only once be of the order of 5-10, a necessary condition for breaking off.

The rather trivial assumption that double envelopes may appear as the result of repeated outbursts of gaseous matter from the central star of a nebula seems unreal (see details in Gurzadian, 1962).

A curious picture of the evolution of planetary nebulae is obtained: every nebula comes into existence as single-enveloped, then becomes double-enveloped, and finally, with the expansion of the nebula, the outer envelope disappears earlier while the basic (first) one obtains a ring-like form in its projection in the sky.

The number of double-enveloped planetary nebulae is rather large, about 30. It will grow with the accomplishment of the technique of photographing faint (but not star-like) planetary nebulae. The universality, for all planetary nebulae, of the phenomenon leading to the birth of second envelopes is becoming obvious.

Under such conditions of frequency of occurrence it is extremely desirable to carry out special experiments to test the basic positions of the above-mentioned theory regarding the formation of double-enveloped nebulae. According to this theory the expansion of the outer envelope must take place with a velocity of about $10 \mathrm{~km} / \mathrm{sec}$ greater than the expansion of the first (inner) envelope.

The measurement of such a difference in the velocities of expansion does not present in itself a major problem, but in an overwhelming majority of cases the brightness of the external envelope is very small compared with the brightness of the basic envelope, wherein lies the difficulty. Nevertheless, I hope that Osterbrock and Münch, with the help of large telescopes, will be able to carry out such work, if only for a few nebulae the outer envelopes of which are sufficiently bright. I have in mind particularly NGC 2392 (which has the greatest known velocity of expansion), NGC 7009, NGC 7662 , and others.

(3) The problem of magnetic fields in planetary nebulae. Bipolarity is a frequent property of the structure of planetary nebulae. It is present in the tendency of a nebula to form two regions with heightened brightness ('cap') set symmetrically in relation to the nucleus. Examples of bipolar nebulae are NGC 2474-5, NGC 7026, anon. $16^{\mathrm{h}} 10^{\mathrm{m}} .5$, A 70, NGC 3587, NGC 7293, and many others.

The circumstance of the bipolar structure being very well defined (always two 'caps' set strictly at opposite directions relative to the nucleus) motivated the present author to suggest ten years ago the hypothesis of the electromagnetic nature of that property. In this manner there appeared the first suggestions of the existence of magnetic fields in planetary nebulae. It is surprising that the supposition of a bipolar magnetic field, having nothing in common with the bipolar magnetic field of the central star, explains 
very well the diversity of structure, external shapes and forms. It appears that the elongation or extension in form of the planetary nebulae is primarily a consequence of the activity of their own bipolar magnetic fields.

I cannot say in what manner the bipolar magnetic fields arise and remain in planetary nebulae. It is indisputable that the bipolar structure cannot exist for long. Their continual existence would require the activity of some continual force. In the basic envelope a magnetic field of the non-pointed dipole-like type (when the dimensions of the dipole are comparable to the dimensions of the nebula) may explain the existence of 'caps'. However, the existence of a chaotic, irregular magnetic field within the central rarefied region of a nebula is not excluded.

These arguments do not, of course, prove the existence of magnetic fields in planetary nebulae of precisely the bipolar type. But we have independently obtained sufficiently convincing evidence of the existence in general, of magnetic fields in planetary nebulae. I have in mind the fact of the radio emission of non-thermal (synchrotron) nature of some planetary nebulae. However, radio observations do not yet say anything about the nature of the magnetic field itself. New ways must be explored in this direction. I do not know in what manner to obtain relevant data on the character of the magnetic fields in planetary nebulae. Perhaps it can be done by radio-polarimetric observations carried out on the relation of planetary nebulae with non-thermal radio emission.

The number of bipolar nebulae is great, constituting almost half of all the planetary nebulae photographed sufficiently well to permit an understanding of their structure. This fact alone is sufficient ground for attracting special attention to the bipolar phenomenon.

Perhaps one of the most characteristic aspects of our symposium is that for the first time we have begun to speak directly about the magnetic fields in planetary nebulae. In this connection I particularly like the report by Menzel, who underlined the necessity of the existence of magnetic fields connected with the nebula, for understanding the peculiar features of its structure. I hope that in the future both theoretical and observational investigations in this direction will make our understanding of the magnetic fields in planetary nebulae more precise.

(4) The problems of gas dynamics. At present we do not have many results on the problems of gas dynamics of planetary nebulae. In this respect we must welcome the initiatives of Kahn, Mathews and others in formulating these interesting problems. It is necessary to continue these investigations, however, using more refined physical assumptions. Perhaps we should be wary of repeating the errors of theoreticians working on the problems of the evolution and internal constitution of stars. We should have in mind that the possibility of checking theory with observations is more likely in the case of gaseous nebulae than in the case of stellar interiors; in any event, we may see the nebulae throughout.

I think that it is necessary to continue theoretical investigations regarding the stability 
of gaseous envelopes in general and the stability of the forms of planetary nebulae in particular, taking into account the action of magnetic fields. Gaseous envelopes, originating around stars by diverse ways (expulsion, outflow, breaking off), may possess various degrees of stability of shape. Considerations about the stability of forms of envelopes may in the end show us the way the nebulae originate.

(5) The problem of condensations in NGC 7293. Two concepts of the formation of condensations in the nebula NGC 7293 - static and dynamic - have existed until recently. The static concept was suggested by Zanstra (1955) and may be regarded as arising in the following manner. Condensations are regions of decreased temperature and consequently increased density of matter in a nebula in pressure equilibrium with the substrate. The condensations themselves have come into being as a result of the energy expended by free electrons in the excitation of the forbidden lines. The dynamic concept, suggested by Gurzadian (1962), connects the origin of condensations wholly with processes of non-stationary character, occurring in the central star of a nebula.

When Daub (1963), in exchange for the strongly idealized model of Zanstra, examined a more realistic model of the nebula with due regard for its chemical composition and the changes of the state of ionization, it appeared that the formation of condensations was simply impossible. The same problem, but from the viewpoint of pure thermal stability of a planetary nebula, was examined also by Field (1965) and Sofia (1966). They have come to the conclusion that within the broad limits of the physical conditions (the dilution factor of the radiation, chemical composition and density in a nebula, the temperature of the nucleus, etc.) the thermal equilibrium is stable, and consequently, the formation of condensations as a result of the disturbance of the thermal stability is impossible.

Thus, Daub, Field and Sofia have shown convincingly the inconsistency of the static concept of the condensations. We have to consider such a conclusion as progress in the problem discussed, for in this way we substantially narrow the field of possibilities. The dynamic nature of the condensations becomes more attractive.

No concrete mechanism of the formation of condensations within the framework of dynamic processes has been suggested. However, the qualitative picture follows. First, the appearance of condensations is to be connected only with the activity of the nucleus of the planetary nebula. They are clots or filaments of gaseous matter ejected or emitted from the nucleus. The velocity of their expulsion considerably increases the velocity of the expansion of the nebula itself.

The very ejection of clots is one of the results of the spontaneous rise in the activity of the nucleus taking place some time, probably some hundreds or thousands of years, previously. Then there was a long period of comparative calm, during which the process of the small-scale corpuscular outflow of charged particles does not stop. When overtaking condensations ejected earlier with great velocity, these particles bring about the phenomenon which we observe. This is similar to the interaction of the corpuscular 
stream of the Sun with the heads of comets. That is how, apparently, one may explain the rather clearly revealed polar forms of those condensations in the photographs of NGC 7293. No calculations have been made to quantitatively test the above hypothesis, it being more advisable to first have some confirmation or refutation of this hypothesis through direct observations. In particular, an attempt should be made to measure the proper motions of these condensations. Mrs Liller has informed us that according to her measurements, the velocity of radial motions of these condensations is about $10 \mathrm{~km} / \mathrm{sec}$, if the distance of this nebula is $200 \mathrm{parsec}$. But this value of the distance is overestimated at least by a factor of 2 or 3 ; the new estimates, obtained by astrophysical methods, give for the distance of NGC 7293 a value of less than 100 parsec, equal to 75 parsec (Gurzadian, 1963). Therefore, it is desirable to reexamine this problem, especially if we have in mind the preliminary character of these measurements, as I have learned from W. Liller.

It is desirable to obtain photographs in various wavelength regions of the filaments with the purpose of obtaining some data about the distribution of energy in their spectrum. Decreases in the brightness in the course of time should be expected for the condensations themselves. It is clear that any result obtained through such observations would clarify our initial assumptions. I would like to emphasize that until now, as far as I know, no special observations have been carried out on these condensations, and it would be very well if the present representatives of observatories having powerful telescopes showed initiative in this work.

Although we have spoken here about the condensations in the nebula NGC 7293 only, it is quite obvious that we are concerned with a general phenomenon. It is possible that such a generalization is premature, in fact, except for NGC 7293 and NGC 6270, since we do not possess any data about the presence of condensations in other planetary nebulae. Moreover, we occasionally come across statements that they do not exist in, e.g., the nebula IC 418 or NGC 7662. This may be the case, for the position of the dynamic concept of the origin of condensations indicates that during certain periods of the life of a nebula they may not exist at all. However, and here one should not hurry, one must bear in mind that the conditions for discovery of the condensations in the case of NGC 7293 are exceptionally favorable primarily due to its being one of the nearest, if not the nearest nebula. The author feels that condensations in planetary nebulae may serve as important and independent indicators of the processes and phenomena taking place in the nuclei of planetary nebulae.

\section{References}

Daub, C.T. (1963) Astrophys. J., 137, 185.

Field, G.B. (1965) Astrophys. J., 142, 531.

Gurzadian, G. (1962) Planetary Nebulae, Moscow.

Gurzadian, G. (1963) Commun. Burakan Obs., 34, 59.

Minin, I. (1958) Problems of Cosmogony, Moscow, p. 211. 
Astrophys. J., 145, 84.

in Vistas in Astronomy, Vol. I, Pergamon Press, London and New York, p. 256.

\section{DISCUSSION}

Kahn: I feel much less optimistic than Gurzadian about the possibility of invoking magnetic-field effects to explain the structures observed in planetary nebulae. In the study of problems involving the structure of the Galaxy or the behaviour of the interstellar gas, people have often tried to give explanations in terms of a magnetic field; but the magnetic field has usually proved rather ineffective. It seems likely that students of planetary nebulae will have a similar experience.

Woyk: As to whether or not it is meaningless to consider magnetic field models in planetaries there is the question of other types of energies which perhaps should be taken into account when drawing limits of the magnetic energy.

Capriotti: J.P. Harrington has made a study of the origin of the condensations as a thermal instability phenomenon. He has taken into account the changing behavior of the nebular radiation field with distance from the central star while in previous studies the assumption was always made that the nebular radiation field had a blackbody spectral distribution. Negative results were obtained in that seemingly unrealistically high stellar-effective temperatures were needed in order to produce thermal instabilities.

In nebulae that are optically thick in the Lyman continuum, the Ly- $\alpha$-radiation pressure may exceed the gas pressure. A realistic study of the origin of the condensations in these objects should include the effects of the work done by $L y-\alpha$ radiation.

Kahn: In essence, the restriction put on the magnetic field by Woltjer's theorem relates the positive energy of the magnetic field laced through the nebula to the negative energy of the nebula in the gravitational field of the central star.

Osterbrock: What do you think the distance of NGC 7293 is?

Gurzadian: I think that the distance of NGC 7293 is nearly 60 parsec, or, in any case, less than 100 parsec.

Liller: I took the distance to NGC 7293 to be 200 parsec simply because I was commenting on Vorontsov-Velyaminov's paper and this is roughly the distance derived by him. I certainly did not intend to imply that this value was sacred. 